\title{
What was lost in translation in the DHA trial is whom you should intend to treat
}

Sally A Frautschy ${ }^{* 1,2}$ and Greg M Cole $e^{1,2}$

\begin{abstract}
The results of a randomized double-blind placebocontrolled trial with docosahexaenoic acid (DHA) supplementation in mild to moderate Alzheimer's disease (AD) published by Quinn and colleagues in JAMA argues against overall efficacy of DHA in slowing progression. However, certain caveats in the results caution against discarding DHA altogether, raising questions about oxidation, dosage, pharmacogenomics and stage of intervention. One potential misconception is that what works for prevention will slow progression in AD subjects. Preclinical studies with DHA supported the rationale for early stage intervention; and three epidemiological studies indicated DHA intake was associated with reduced risk in non-apolipoprotein E4 (ApoE4) carriers. Putative drugs are initially tested for impact on progression because prevention approaches are problematic. However, should a drug be discarded for prevention if it fails to modify progression? Consistent with epidemiology, DHA significantly benefited two measures of cognition in mild to moderate nonApoE4 carriers. Although the results of this trial were overall negative, failing to modify other outcomes, this commentary discusses important questions raised by them. Should future trials pursue DHA in non-ApoE4 carriers for slowing progression? Since in vivo oxidation of DHA may have adverse effects, particularly in ApoE4 patients, should preclinical and clinical studies be performed to optimize dose and mitigate oxidation before pursuing intervention or prevention trials with DHA? And finally, should DHA be tested now for mild cognitive impairment or prevention?
\end{abstract}

*Correspondence: frautsch@ucla.edu

${ }^{2}$ Departments of Medicine and Neurology, Mary S Easton Center for Alzheimer's Disease Research at UCLA, 10911 Weyburn Avenue, Suite 200 Los Angeles CA 90095, USA

Full list of author information is available at the end of the article
Subjects with baseline mini-mental state examination (MMSE) scores of mild to moderate Alzheimer's disease (AD) were treated with algal docosahexaenoic acid (DHA, 2,000 mg/day) for 18 months in a randomized double-blind placebo-controlled trial to determine the impact on $\mathrm{AD}$ progression. The rationale for testing DHA was strong. It is enriched in neuronal membranes but depleted in AD. Multiple epidemiological studies report diets rich in fish or DHA reduce AD risk, most clearly in non-apolipoprotein E4 (ApoE4) carriers [1]. Preclinical studies with DHA have not yet modeled ApoE isoform pharmacogenomics, but mice transgenic for familial dominant $\mathrm{AD}$ mutations that elevate $\beta$-amyloid $(\mathrm{A} \beta)$ production are vulnerable to dietary DHA depletion. DHA and its metabolites pleiotropically impact $A \beta$ production, insulin/neurotrophic signaling, tau kinase activation and synaptic plasticity [1]. Although DHA had no impact on cognitive or functional decline based on intent to treat $\mathrm{AD}$, in non-ApoE4 carriers, DHA supplementation appeared to reduce declines in MMSE and Alzheimer Disease Assessment Scale-Cognitive (ADASCog). This commentary discusses the trial's results and important questions raised, including the need for optimization of dose and antioxidant combinations and whether there should be further investigation of the impact of DHA on slowing cognitive decline in nonApoE4 carriers. Perhaps a larger issue is whether agents directed at amyloid or tau pathology or associated with reduced $\mathrm{AD}$ risk should be translated with an intent-totreat earlier disease stages rather than mild to moderate AD.

The randomized trial of DHA for AD provides evidence that DHA supplementation provides no general benefit to $\mathrm{AD}$ patients, including no overall impact on Clinical Dementia Rating (CDR) scale, ADAS-Cog, MMSE, Neuropsychiatric Inventory (NPI; $P=0.11$ ) and Activities of Daily Living (ADL) [2]. The authors argue that DHA may still have potential for prevention. Thus, a fundamental question arising out of this study is the stage at which we should treat. First, three smaller studies showed an apparent benefit from fish oil treatment in mild cognitive impairment but not in mild to moderate $\mathrm{AD}$ subjects [3-5]. With age-associated memory 
impairment, one small trial [6] and the larger 485-subject MIDAS (Memory Improvement with DHA Study) trial [7] found significant cognitive benefits with DHA. While there have been two fish oil trials in unimpaired elderly in which no cognitive benefits were observed [8,9], subjects in both were cognitively normal at baseline, and the latter failed to show significant cognitive decline in the placebo group. This study argues that fish oil is not a cognitive enhancer, but does not examine disease modification in subjects with pathology-driven memory deficits. Second, animal studies report DHA/fish oil act on two pathological endpoints that plateau by mild to moderate stages: $A \beta$ accumulation [10] and loss of superior cortical drebrin, an excitatory synaptic marker [11] (reviewed in [1]). Dramatic medial and superior temporal drebrin loss plateaus early with mild cognitive impairment by MMSE 26 [12], so loss has already occurred in trial subjects. While DHA reduced both $A \beta$ and tau pathology in 3xTg $\mathrm{AD}$ mice [13], that intervention was early (prepathology). In contrast, with late post-pathology intervention in human tau transgenic mice with significant neuron loss, we find DHA treatment is insufficient to produce significant cognitive and synaptic improvements (GMC and SAF, Society for Neuroscience presentations, 2010). Finally, epidemiological risk factors may be relevant to prevention, but not necessarily to treatment. Animal model data with DHA support early intervention for primary prevention or mild cognitive impairment and suggest a failure to impact tangle and neuron loss driven deficits at later stages.

Although the data demonstrate that DHA has no general benefit for $\mathrm{AD}$, a concern remains as to whether the key negative effect may be driven by the failure of ApoE4 subjects to respond. Since non-ApoE4 carriers comprise a large segment of the US (approximately 75\%) and AD (approximately 50\%) populations, whether DHA may slow progression in non-ApoE4 carriers is important. Figure 3 in [2] indicates that $40 \%$ of non-ApoE4 carriers showed significant $(P=0.03)$ stabilization of both ADAS-Cog and MMSE, but not with correction for multiple comparisons. The authors point out that three epidemiological studies showed reduced risk with fish consumption only in the ApoE4 non-carriers, but add that pharmacogenomic interaction was not seen with CDR, ADL or NPI. For example, NPI showed a trend independent of genotype, worsening less (2.93 points) in the DHA group than in the placebo group (5.09 points, $P=0.11)$. Are pathogenic mechanisms impacting NPI, ADL, CDR and MMSE/ADAS-Cog the same? Thus, any pharmacogenomic potential of DHA requires clarification.

For prevention or treatment, one might expect ApoE genotype-DHA interactions. Because ApoE4 accelerates pathogenesis, age-matched ApoE4 patients may have more intractable AD pathology. Further, one important target of DHA is insulin resistance [14], but drugs targeting insulin resistance (insulin or peroxisome proliferatoractivated receptor (PPAR) $\gamma$ agonists) appears more effective at reducing cognitive deficits in ApoE3 carriers than ApoE4 carriers [15]. ApoE is a major central nervous system lipid transport protein with isoform-dependent trafficking likely to impact DHA compartmentalization in the brain. Finally, ApoE4 increases oxidative stress, and with six double bonds, DHA is readily oxidized.

This raises other critical issues that need to be addressed before pursuing a future trial: dose and oxidation. The authors discuss the need to investigate potential combinations of DHA with antioxidants in AD patients, given apparent benefits with combinations of fish oil and lutein or lipoate in small trials and with antioxidants in the Souvenaid trial. Oxidation of DHA to neuroprostanes is associated with synaptic loss. Further oxidation produces a toxic end-product, 4-hydroxyhexenal, that contributes to neuron death and defective uptake of glucose by neurons and glutamate by astrocytes. Clinical studies demonstrate that similar dosing with marine n-3 fatty acids (polyunsaturated fatty acids with a double bond at the third carbon), including DHA, can deplete vitamin $E$ and increase some peripheral measures of oxidative damage, particularly with dosing up to 6 months [16]. Because DHA is enriched in the brain where oxidative damage is already increased in $\mathrm{AD}$ patients, antioxidant supplements optimized for AD brain appear crucial. Even though marine n-3 fatty acids can deplete vitamin E, high dose vitamin E (900 IU) did not reduce measures of lipid peroxidation in human plasma [17], so vitamin E supplementation is probably not sufficient. In mice the lipophilic phenolic antioxidant food additive butylhydroxytoluene attenuated measures of lipid peroxidation in plasma after high intake of fish oil [18]. The preclinical studies with DHA in AD mouse models require encapsulation of DHA in the chow to minimize oxidation $[10,11]$. Also, using the US Food and Drug Administration's equation to estimate the human equivalent doses, the clinical trial dose was three-fold higher than the efficacious preclinical dose in mice $[10,11]$, and twice as high as in the MIDAS trial [7], raising questions about whether the dose may have been too high, potentially exacerbating oxidative damage.

Possible cognitive benefits in patient subgroups (pharmacogenomic or otherwise) would be strengthened by evidence of a biomarker response, arguing for the need to validate neuroimaging, cerebrospinal fluid or plasma biomarker responses in preclinical studies going forward. MRI was performed in a small subset of subjects, showing that volumentrics of the left hippocampus in the DHA group showed trends to be smaller than in the placebo group $(P=0.17)$, which may indicate brain shrinkage. In the AN1792 active $A \beta$ vaccination, 
MRI shrinkage was attributed to plaque clearance. Since drugs may only work in a subset of patients, it would be helpful in large studies where neuroimaging or cerebrospinal fluid biomarker analysis are less feasible to identify likely responders with plasma biomarkers. A difficult task at hand is to design future DHA or other trials with earlier intervention to include validated surrogate and/or diagnostic biomarkers that have shown DHA responses in animal models. For tracking adverse effects of DHA, it is important to measure blood vitamin E depletion and lipid peroxides (thiobarbituric acid reactive substances, malondialdehyde, or the specific byproduct of DHA oxidation, 4-hydroxyhexenal). Biomarker validation could track mechanisms and lower trial costs and facilitate choice of efficacious doses before proceeding to longer term, more costly trials to evaluate conversion to $\mathrm{AD}$.

\section{Conclusion}

The study by Quinn and colleagues provides additional rationale to test DHA for prevention, with focus on nonApoE4 carriers, but problems with DHA dosing and oxidation need to be addressed (particularly if an antioxidant could correct a failed ApoE4 response to DHA). Additional preclinical studies of stage-dependent efficacy and ApoE4-DHA interaction may help to clarify whether ApoE genotype affects outcomes and how this can be mitigated, possibly with antioxidants or nonsteroidal anti-inflammatory drugs (NSAIDs). Beyond pharmacogenomic roadblocks emerging with DHA and other interventions, all of the epidemiology and most of the animal model data that have been generated are most relevant to early stage interventions, but have been translated in clinical trials in mild to moderate $\mathrm{AD}$, potentially resulting in an intent-to-treat the wrong group. The pre-clinical conclusions may not be wrong, but simply still lost in this translation.

\section{Abbreviations}

A $\beta, \beta$-amyloid; AD, Alzheimer's disease; ADAS-Cog, Alzheimer Disease Assessment Scale-Cognitive; ADL, Activities of Daily Living; ApoE, apolipoprotein E; CDR, Clinical Dementia Rating; DHA, docosahexaenoic acid; MIDAS, Memory Improvement with DHA Study; MMSE, mini-mental state examination; MRI, magnetic resonance imaging; NPI, Neuropsychiatric Inventory.

\section{Competing interests}

GMC has received reimbursements from Martek Biosciences for travel and lectures that he has presented on DHA and as a member of their expert panel. SAF has no competing financial interests

\section{Authors' contributions}

GMC and SAF made equal contributions in writing this commentary.

\section{Author information}

GMC is Associate Director of the Mary S Easton University of California, Los Angeles (UCLA) Alzheimer's Disease Research Center and of the Veteran's Greater Los Angeles Healthcare System, Geriatric Research Education and Clinical Core (GRECC). SAF is Chief of Neurogerontology at the GRECC, and both SAF and GMC are Professors of Medicine and Neurology at UCLA. GMC and SAF have contributed to the field in elucidating mechanisms in Alzheimer's and developing models for translation.

\section{Acknowledgments}

We thank the Mary S Easton Alzheimer's Drug Discovery Program and VA Merits for funding (SAF, GMC) and NIH R01AG13471 (GMC), NIH R01AT03008 (GMC), NIH R01AG021795 (SAF), NIH U01AG028783 (SAF), NIH RC1AG035878 (SAF, GMC).

\section{Author details}

'Veterans Greater Los Angeles Healthcare System, Geriatric Research Education and Clinical Center, 16111 Plummer St, North Hills, CA 91343, USA. ${ }^{2}$ Departments of Medicine and Neurology, Mary S Easton Center for Alzheimer's Disease Research at UCLA, 10911 Weyburn Avenue, Suite 200 Los Angeles CA 90095, USA.

Published: 20 January 2011

\section{References}

1. Cole GM, Frautschy SA: DHA may prevent age-related dementia. J Nutr 2010, 140:869-874

2. Quinn JF, Raman R, Thomas RG, Yurko-Mauro K, Nelson EB, Van Dyck C, Galvin JE, Emond J, Jack CR Jr, Weiner M, Shinto L, Aisen PS: Docosahexaenoic acid supplementation and cognitive decline in Alzheimer disease: a randomized trial. JAMA 2010, 304:1903-1911.

3. Freund-Levi Y, Eriksdotter-Jonhagen M, Cederholm T, Basun H, Faxen-Irving G, Garlind A, Vedin I, Vessby B, Wahlund LO, Palmblad J: Omega-3 fatty acid treatment in 174 patients with mild to moderate Alzheimer disease: OmegAD study: a randomized double-blind trial. Arch Neurol 2006, 63:1402-1408.

4. Chiu CC, Su KP, Cheng TC, Liu HC, Chang CJ, Dewey ME, Stewart R, Huang SY: The effects of omega-3 fatty acids monotherapy in Alzheimer's disease and mild cognitive impairment: a preliminary randomized double-blind placebo-controlled study. Prog Neuropsychopharmacol Biol Psychiatry 2008, 32:1538-1544.

5. Kotani S, Sakaguchi E, Warashina S, Matsukawa N, Ishikura Y, Kiso Y, Sakakibara M, Yoshimoto T, Guo J, Yamashima T: Dietary supplementation of arachidonic and docosahexaenoic acids improves cognitive dysfunction. Neurosci Res 2006, 56:159-164

6. Johnson EJ, MCDonald K, Caldarella SM, Chung HY, Troen AM, Snodderly DM: Cognitive findings of an exploratory trial of docosahexaenoic acid and lutein supplementation in older women. Nutr Neurosci 2008, 11:75-83.

7. Yurko-Mauro K, McCarthy D, Rom D, Nelson EB, Ryan AS, Blackwell A, Salem N $\mathrm{Jr}$, Stedman M, Investigators M: Beneficial effects of docosahexaenoic acid on cognition in age-related cognitive decline. Alzheimers Dement 2010, 6:456-464.

8. Van de Rest O, Geleijnse J, Kok F, van Staveren W, Dullemeijer C, OldeRikkert $M$, Beekman A, de Groot L: Effect of fish oil on cognitive performance in older subjects: a randomized controlled trial. Neurology 2008, 71:430-438.

9. Dangour AD, Allen E, Elbourne D, Fasey N, Fletcher AE, Hardy P, Holder GE, Knight R, Letley L, Richards M, Uauy R: Effect of 2-y n-3 long-chain polyunsaturated fatty acid supplementation on cognitive function in older people: a randomized, double-blind, controlled trial. Am J Clin Nutr 2010, 91:1725-1732.

10. Lim GP, Calon F, Morihara T, Yang F, Teter B, Ubeda O, Salem N Jr, Frautschy SA, Cole GM: A diet enriched with the omega-3 fatty acid docosahexaenoic acid reduces amyloid burden in an aged Alzheimer mouse model. J Neurosci 2005, 25:3032-3040.

11. Calon F, Lim GP, Yang F, Morihara T, Teter B, Ubeda O, Rostaing P, Triller A, Salem N Jr, Ashe KH, Frautschy SA, Cole GM: Docosahexaenoic acid protects from dendritic pathology in an Alzheimer's disease mouse model. Neuron 2004, 43:633-645.

12. Counts SE, Nadeem M, Lad SP, Wuu J, Mufson EJ: Differential expression of synaptic proteins in the frontal and temporal cortex of elderly subjects with mild cognitive impairment. J Neuropathol Exp Neurol 2006, 65:592-601.

13. Green KN, Martinez-Coria H, Khashwji H, Hall EB, Yurko-Mauro KA, Ellis L, LaFerla FM: Dietary docosahexaenoic acid and docosapentaenoic acid ameliorate amyloid-beta and tau pathology via a mechanism involving presenilin 1 levels. J Neurosci 2007, 27:4385-4395.

14. Ma QL, Yang F, Rosario ER, Ubeda OJ, Beech W, Gant DJ, Chen PP, Hudspeth B, 
Chen C, Zhao Y, Vinters HV, Frautschy SA, Cole GM: Beta-amyloid oligomers induce phosphorylation of tau and inactivation of insulin receptor substrate via c-Jun $\mathrm{N}$-terminal kinase signaling: suppression by omega-3 fatty acids and curcumin. J Neurosci 2009, 29:9078-9089.

15. Reger MA, Watson GS, Green PS, Baker LD, Cholerton B, Fishel MA, Plymate SR, Cherrier MM, Schellenberg GD, Frey WH, 2nd, Craft S: Intranasal insulin administration dose-dependently modulates verbal memory and plasma amyloid-beta in memory-impaired older adults. J Alzheimers Dis 2008, 13:323-331.

16. Palozza P, Sgarlata E, Luberto C, Piccioni E, Anti M, Marra G, Armelao F, Franceschelli P, Bartoli GM: n-3 fatty acids induce oxidative modifications in human erythrocytes depending on dose and duration of dietary supplementation. Am J Clin Nutr 1996, 64:297-304.
17. Allard JP, Kurian R, Aghdassi E, Muggli R, Royall D: Lipid peroxidation during n-3 fatty acid and vitamin E supplementation in humans. Lipids 1997, 32:535-541.

18. Gonzalez MJ, Gray Jl, Schemmel RA, Dugan L Jr, Welsch CW: Lipid peroxidation products are elevated in fish oil diets even in the presence of added antioxidants. J Nutr 1992, 122:2190-2195.

doi:10.1186/alzrt61

Cite this article as: Frautschy SA, Cole GM: What was lost in translation in the DHA trial is whom you should intend to treat. Alzheimer's Research \&

Therapy 2011, 3:2. 